\title{
Protokoll der Generalversammlung in Innsbruck
}

(Auszug, Mitglieder können im Memberbereich der ÖGUM-Website die Komplettversion einsehen)

Donnerstag, 30.10.2014, 17:45 - 18:30 Uhr; Congress Innsbruck, Saal Brüssel Beginn: 17:47 Uhr; Anwesend: (55 Mitglieder)

1. Begrüßung und Feststellung der Beschlussfähigkeit

H. Steiner begrüßt die Teilnehmer/Innen.

Da die Generalversammlung erst beschlussfähig ist, wenn mindestens die Hälfte der wahlberechtigten Mitglieder anwesend ist, wird statutengemäß nach einigen Minuten die Generalversammlung nochmals eröffnet. Die Beschlussfähigkeit kann somit festgestellt werden.

2. Genehmigung des Protokolls der Generalversammlung vom 11.10.2013 Das Protokoll wird einstimmig angenommen.

3. Bericht des Präsidenten $\mathrm{H}$. Steiner stellt die Mitgliederzahl in den Fachbereichen der ÖGUM vor. Er betont die Wichtigkeit der Mitarbeit in den einzelnen Arbeitskreisen und lädt die Mitglieder ein, in diesen aktiv zu werden.

Die Zertifizierung der Notfallsonographie ist abgeschlossen. H. Steiner bedankt sich bei G. Mathis für die Ausarbeitung des Konzeptes.

H. Steiner spricht den Rücktritt von S. Nöldeke als DEGUM-Präsident an. Er hofft, dass sich die Turbulenzen legen und auf einen guten Neuanfang. Die EFSUMB-Beiträge wurden, laut Beschluss der Vorstände im Mai 2014, von $€ 8$ auf $€ 7$ reduziert. Die Reduktion ging in erster Linie von der DEGUM aus, die eine Adaption des EFSUMB-Beitrages anstrebte. H. Steiner bedankt sich bei A. Klauser für die sehr gute Organisation des 38 .
Dreiländertreffens und weist auf den nächsten Ultraschallkongress in Österreich hin, der 2017 in Linz stattfinden wird.

4. Bericht des Kassiers

C. Kollmann stellt den Finanzbericht des Jahres 2013 vor. Die Zahlungsmoral von $96,7 \%$ ist sehr zufriedenstellend. Die Mitgliedsgebühren werden für 2015 nicht erhöht.

5. Entlastung des Vorstandes ÖGUM-Rechnungsprüfer T. Ybinger bestätigt die korrekte Finanzgebarung des Vereins nach dem österr. Vereinsgesetz für den Prüfungszeitraum 01.01.2013-31.12.2013, sowie die ordnungsgemäße Verwendung der Vereinsmittel laut den Vereinsstatuten. Die Belege wurden stichprobenartig geprüft.

G. Mostbeck stellt den Antrag auf Entlastung des Vorstands, welcher einstimmig angenommen wird.

$\mathrm{H}$. Steiner bedankt sich im Namen des Vorstandes für das entgegengebrachte Vertrauen.

6. Berichte der Landesgruppen Folgende Landesgruppen haben Berichte vorgetragen:

Vorarlberg: ÖGUM/ÖGIM-Kurse wurden abgehalten;

Tirol: ÖGUM-Kurse wurden abgehalten, vorwiegend im MSK-Bereich, Abdomensonografiekurse sind geplant; Salzburg: Kurse wurden abgehalten, weitere Kurse werden stattfinden; Oberösterreich: Kurse wurden abgehalten; Frage zur Notwendigkeit der quartalsmäßigen Übermittlung der Kontoauszüge des Landesgruppenkontos an die Geschäftsstelle wird gestellt.

B. Kasperak befürwortet dieses Vorgehen, da dies eine Erleichterung für den Kassier darstellt. C. Kollmann findet es wichtig, da er damit eine Übersicht erhaltet, die ihm die Arbeit als Kassier erleichtert.

Burgenland: Neue Leitung der Landesgruppe. Vorsitzende OÄ Dr. Maria Feike, Stellvertreter:

OA Dr. Josef Weiss

Steiermark: Leitung wird im Herbst neu gewählt. Stolzalpe Kurse sollen in Zukunft ÖGUM-zertifiziert werden; Kärnten: Leitung wird neu gewählt. Wien: Im Dezember wird die Generalversammlung mit Neuwahlen stattfinden. Genauere Informationen zu den Aktivitäten der Landesgruppen sind auf der ÖGUM Website abrufbar.

7. Thieme Zeitschrift „Ultraschall in der Medizin“

$H$. Steiner spricht das gemeinsame Vorgehen der drei Gesellschaften bezüglich der Kündigung des Thieme Vertrages an. Von Seiten der DEGUM wurden schon länger Diskussionen mit dem Verlag geführt, mit dem Ziel Kosten einzusparen und Thieme zu überzeugen, modernere Strukturen anzubieten. Leider ergaben diese Diskussionen keine zufriedenstellende Lösung.

Die Kündigung der drei Gesellschaften wurde am Treffen der Vorstände im Mai 2014 in Seefeld beschlossen. H. Steiner schildert, dass auf das Kündigungsschreiben der ÖGUM, welches im Juni dieses Jahres an den Thieme Verlag ging, erst vor zwei Wochen reagiert wurde. Im Dezember wird es ein Meeting mit den drei Gesellschaften und den Vertretern des Verlages geben. G. Mostbeck merkt an, dass hier ein massives Kommunikationsproblem vorlag. Es fanden auch keine Gespräche mit den Herausgebern statt. Eine Neugründung mit open Access, einer online Ausgabe und einem Fortbildungsteil wird angestrebt. Den Dialog zwischen den Gesellschaften, dem Verlag und den Herausgebern neu zu starten wäre sehr wichtig. H. Steiner präsentiert die Auswertung der Umfrage die im April dieses Jahres unter den ÖGUM-Mitgliedern stattgefunden hat. Ca. $10 \%$ der ÖGUM-Mitglieder haben an der Umfrage teilgenommen, die Mitglieder tendieren zu einer Print- und OnlineAusgabe.

G. Mostbeck weist darauf hin, dass dringend Reviewer benötigt werden 
und lädt die Arbeitskreise ein, aktiv zu werden.

8. ÖGUM Förderpreis

H. Steiner merkt an, dass 2014 kein Förderpreis vergeben wurde, da die einzige Arbeit die eingereicht wurde, nicht den Richtlinien entsprach. Der ÖGUM-Förderpreisträger 2012, C. Dejaco, präsentiert seine Arbeit mit dem Titel „Sonoelastographie zur Objektivierung der Speicheldrüsenfunktion bei Patienten mit Sjögren Syndrom".

9. Neuwahlen ÖGUM Vorstand H. Steiner präsentiert den Wahlvorschlag für den ÖGUM-Vorstand. B. Pertl stellt sich den Mitgliedern vor. Die Wahl des Vorstandes wird statutenkonform geheim abgehalten. E. Hafner fungiert als Wahlleiter. Von den 49 abgegebenen Wahlzetteln sind 47 mit "JA“ und 2 ungültige Stimmen. Somit ist die erforderliche 2/3-Mehrheit erreicht.

Der neue Vorstand der ÖGUM setzt

sich wie folgt zusammen:

> Präsidentin: Univ. Doz. Dr. Andrea Sabine Klauser

- Präsident-elect: Prim. Dr. Hermann Kathrein

> Past-Präsident: Univ. Prof. Dr. Horst Steiner

- Sekretärin: Prof. Dr. Barbara Pertl Kassier: Ass. Prof. Dr. Christian Kollmann

- weiteres Mitglied: Prof. PD Dr. Helmut Prosch

> kooptiertes Mitglied: Dr. Doris Kerö $\mathrm{H}$. Steiner bedankt sich für die gute $\mathrm{Zu}$ sammenarbeit und übergibt an Präsidentin A. Klauser

A. Klauser bedankt sich bei $\mathrm{H}$. Steiner und hebt nochmals seinen Einsatz für die ÖGUM hervor.

10.Arbeitskreise

A. Klauser stellt die Neukonstitionierung der Arbeitskreise vor. Sie betont die Wichtigkeit der Mitarbeit der Mitglieder in den Arbeitskreisen und lädt dazu ein, in diesen aktiv zu werden.

- Abdomen:

Arbeitskreisleiter: T. Ybinger, Arbeitskreisstellvertreter: W. Sturm

- Allgemeinmedizin:

Arbeitskreisleiter: K. Somavilla, Arbeitskreisstellvertreter: M. Somavilla

- Bewegungsapparat:

Arbeitskreisleiter: C. Schueller-Weidekamm, Arbeitskreisstellvertreter: A. Loizides
Echokardiographie:

Arbeitskreisleiter: D. Kerö, Arbeits-

kreisstellvertreter: G. Huber

- Gefäße:

Die offizielle Wahl in diesem Ar-

beitskreis hat noch nicht stattgefunden.

Wahlvorschlag: W.Sturm und R. Mathies. Das Wahlergebnis wird bekanntgegeben.

- Gynäkologie:

Arbeitskreisleiter: E. Hafner, Arbeitskreisstellvertreter: P. Klaritsch

> Pädiatrie:

Arbeitskreisleiter: M. Riccabona, Arbeitskreisstellvertreter: $\mathrm{G}$.

Schweintzger

- Kopf/Hals:

Die offizielle Wahl in diesem Ar-

beitskreis hat noch nicht stattgefunden. Die Arbeitskreissitzung ist auf morgen verschoben worden. Wahlvorschlag: G. Zettinig und S. Meng.

Das

Wahlergebnis wird bekanntgegeben.

- Mammasonographie:

Arbeitskreisleiter: C. Weismann, Arbeitskreisstellvertreter: M. Daniaux

> Notfallsonographie:

Arbeitskreisleiter: G. Mathis, Ar-

beitskreisstellvertreter: P. Zechner

- Perioperative Sonographie:

Arbeitskreisleiter: H. Koinig, Arbeitskreisstellvertreter: B. Bacher

> Technik \& Qualitätssicherung:

Arbeitskreisleiter: C. Kollmann, Arbeitskreisstellvertreter: T. Ybinger

Thorax:

Arbeitskreisleiter: H. Prosch, Arbeitskreisstellvertreter: $\mathrm{G}$. Mathis

- Urologie:

Arbeitskreisleiter: F. Frauscher, Arbeitskreisstellvertreter: M. Mitterberger

- Nervensonographie:

Dieser Arbeitskreis wurde neu gegründet.

Arbeitskreisleiter: G. Bodner, Ar-

beitskreisstellvertreter: D. Lieba-Samal

11.Allfälliges

R. Faschingbauer fragt an, ob ein Pool an Phantomen über die ÖGUM angeschafft werden könnte.

C. Kollmann spricht die Finanzierung dieses Vorhabens an. H. Steiner merkt an, dass es vorrangig wichtig wäre, den Bedarf solcher Phantome zu erheben.

Ende: 18:30 Uhr:

PDF unterMember-Bereich:

www.oegum.at 